\title{
Antibiotic prescribing practice and adherence to guidelines in primary care in the Cape Town Metro District, South Africa
}

\author{
J Gasson, ${ }^{1}$ BMBS, BMedSci; M Blockman, ${ }^{2}$ MB ChB, BPharm, PG Dip (Health Research Ethics), MMed; B Willems, ${ }^{3}$ MB ChB, DA (SA), FCPHM \\ ${ }^{1}$ Improving Global Health Fellow, Health Impact Assessment, Western Cape Department of Health, South Africa; NHS Thames Valley and \\ Wessex Leadership Academy, UK; THET/UK Aid in association with Health Education England, UK \\ ${ }^{2}$ Department of Internal Medicine, Division of Clinical Pharmacology, Faculty of Health Sciences, University of Cape Town and Groote Schuur \\ Hospital, Cape Town, South Africa \\ ${ }^{3}$ Division of Health Systems and Public Health, Faculty of Medicine and Health Sciences, Stellenbosch University, Cape Town, South Africa
}

Corresponding author: J Gasson (julia.gasson@gmail.com)

Background. Knowledge of antibiotic prescribing practice in primary care in South Africa is limited. As $80 \%$ of human antibiotic use is in
primary care, this knowledge is important in view of the global problem of antibiotic resistance.
Objectives. To assess antibiotic prescribing in primary care facilities in the Cape Town Metro District and compare it with current national
guidelines, and to assess the reasons why prescriptions were not adherent to guidelines.
Methods. A retrospective medical record review was performed in April/May 2016. Records of all patients seen over 2 days in each of
eight representative primary care facilities in the Cape Town Metro District were reviewed. The treatment of any patient who raised a new
complaint on either of those days was recorded. Prophylactic antibiotic courses, tuberculosis treatment and patients with a non-infection
diagnosis were excluded. Treatment was compared with the Standard Treatment Guidelines and Essential Medicines List for South Africa,
Primary Healthcare Level, 2014 edition.
Results. Of 654 records included, $68.7 \%$ indicated that an antibiotic had been prescribed. Overall guideline adherence was $45.1 \%$.
Adherence differed significantly between facilities and according to the physiological system being treated, whether the prescription was
for an adult or paediatric patient, and the antibiotic prescribed. Healthcare professional type and patient gender had no significant effect on
adherence. The main reasons for non-adherence were an undocumented diagnosis (30.5\%), antibiotic not required (21.6\%), incorrect dose
(12.9\%), incorrect drug (11.5\%), and incorrect duration of therapy (9.5\%).
Conclusions. This study demonstrates poor adherence to guidelines. Irrational use of antibiotics is associated with increased antibiotic
resistance. There is an urgent need to improve antibiotic prescribing practice in primary care in the Cape Town Metro District.
SAfr Med J 2018;108(4):304-310. DOI:10.7196/SAMJ.2018.v108i4.12564

The inappropriate and irrational use of antibiotics has contributed significantly to the development of antibiotic resistance. ${ }^{[1-3]}$ Patients infected with resistant organisms have an increased risk of poor clinical outcomes, including death, and consume more healthcare resources. ${ }^{[2]}$ Globally 700000 people currently die each year from drug-resistant infections, and if there is no international action, a scenario analysis has suggested that by the year 2050 this will increase to 10 million deaths annually, with a cumulative cost to global economic output predicted to be USD100 trillion. ${ }^{[2]}$ In a postantibiotic era, infections will become untreatable.

Individual prescribing decisions affect the population level of resistance. Antibiotic resistance in an individual patient is evident for 1 month following an antibiotic course and can last for up to 12 months. ${ }^{[4]}$ There is robust evidence to support prescribing the fewest number of antibiotic courses for the shortest possible period of time. ${ }^{[2-4]}$ Antibiotic stewardship (AS) aims to optimise antibiotic use and limit the development of resistant bacteria while ensuring patient safety. ${ }^{[5]}$ One aspect of AS is the creation and use of evidence-based guidelines to support appropriate antibiotic use.

Eighty percent of antibiotics prescribed for human use are in primary care. ${ }^{[6]}$ Adherence to guidelines in primary care has been shown to vary significantly depending on the condition being treated. ${ }^{[7-13]}$
Studies have shown poor guideline adherence for respiratory problems, ${ }^{[7-10]}$ urinary tract infections, ${ }^{[11,12]}$ tonsillitis ${ }^{[13]}$ and acute diarrhoea. ${ }^{[10]}$ Other variables associated with poor adherence include seniority of the clinician, ${ }^{[14]}$ prescriber workload, ${ }^{[15]}$ patient age ${ }^{[8,14]}$ and area-level socioeconomic status. ${ }^{[8]}$ Using antibiotics incorrectly and inappropriately by not following guidelines can contribute to the development of resistance, as well as poor clinical outcomes including patient morbidity. ${ }^{[1-3]}$

There has been limited published research and evaluation of antibiotic prescribing in primary care in South Africa (SA). A retrospective drug utilisation study carried out in SA in $2010^{[16]}$ reported the total amount of each type of antibiotic prescribed over a year, but unfortunately not the indication or appropriateness. Two pilot projects assessing antibiotic use and resistance were carried out in Brits, North West Province, and Durban, KwaZulu-Natal Province, between 2003 and 2005. ${ }^{[17]}$ They reported the percentage of prescriptions containing an antibiotic, and one site reported on adherence to the Standard Treatment Guidelines included in the South African Essential Medicines List. Neither site commented on the reason for prescription of the antibiotic or the reason for nonadherence. A further study in 2001 evaluated antibiotic prescriptions in nine private primary care facilities across SA. ${ }^{[18]}$ Most of the 
diagnoses for which an antibiotic was prescribed were respiratory conditions, including viral influenza, acute bronchitis, the common cold, cough and acute sinusitis. There has been no comparison in the literature of appropriate prescribing between different healthcare professionals (doctors, nurses, dentists and primary care drug therapist pharmacists) in the public primary healthcare system in Cape Town.

The objectives of SA's National Strategy Framework for antimicrobial resistance $2014-2024,{ }^{[5]}$ the SA Antibiotic Stewardship Programme, the national Best Care Always Campaign and the World Health Organization (WHO) ${ }^{[1,19]}$ all support increasing understanding of prescribing practice. However, with the lack of recent research it remains unclear what current antibiotic prescribing practice is in primary care. The Standard Treatment Guidelines and Essential Medicines List for South Africa, Primary Healthcare Level, 2014 edition (EML), ${ }^{[20]}$ provides a comprehensive, evidence-based guideline for prescribing, specifically in primary care, but the degree of adherence to it is unknown. Increased understanding will enable AS policy and interventions to be appropriately directed to optimise antibiotic use and potentially minimise antibiotic resistance.

\section{Objectives}

To assess current antibiotic prescribing practice in primary care facilities in the Cape Town Metro District, SA, and compare it with current national guidelines, and to assess the reasons why prescriptions were not adherent to guidelines.

\section{Methods}

A retrospective medical record review was performed. In the Cape Town Metro District, there are 152 public primary healthcare facilities in eight subdistricts. Nine community health centres (CHCs) and 40 community day centres (CDCs) are managed by the Western Cape Provincial Government, and the other clinics, satellite clinics and mobile clinics are run by the City of Cape Town municipality. ${ }^{[21]}$ The city and the province have separate management teams and governance structures. To avoid complexity, this study looked only at the CDCs and CHCs. One CHC or CDC was selected at random from each of the eight subdistricts using an Excel random number tool to give a representative sample of the district. For reporting purposes, the facilities were renamed clinics A to $\mathrm{H}$.

Data were collected on site from medical records by the primary investigator (JG) using an electronic collection tool. Two days were spent in each facility during April or May 2016. Records were made of those patients who attended with a new problem during either of the two study days or who attended for a chronic or routine review appointment and raised an acute issue that day. Data recorded included patient gender and age, the type of healthcare professional who saw the patient, the diagnosis documented, and details about any antibiotic prescription given.

Only treatment antibiotics were recorded. Prophylactic antibiotics and tuberculosis treatment were excluded. Patients with a documented diagnosis were divided into those with and without an infection. Those without an infection were excluded from analysis. If a specific diagnosis was not recorded, the diagnosis was categorised as 'unknown. Only patients with a definite infection or an unknown diagnosis were included in the analysis.

Paediatric patients were defined as aged 0 - 16 years inclusive, and adult patients as aged $\geq 17$ years. Each patient was also categorised by the physiological system relating to their diagnosis. If a diagnosis was not recorded, the system category was determined by the main presenting symptom documented.
For all cases of infection or possible infection, the prescription or lack of prescription of an antibiotic ('non-prescription') was compared with the management advised in the 2014 EML for primary care. ${ }^{[20]}$ The case was deemed adherent if a diagnosis was made, the indication to prescribe an antibiotic or not was correct, and if applicable, if every aspect of the prescription (choice of antibiotic, dose, duration and frequency per day) was also correct. Data were analysed using Excel 2010 (Microsoft, USA) and Stata 13 (StataCorp, USA).

Ethics approval was granted by the University of Cape Town Faculty of Health Sciences Human Research Ethics Committee on 11 March 2016 (ref. no. 138/2016).

\section{Results}

The clinical records of 654 patients were included in the analysis (see Fig. 1 for inclusion and exclusion of patient notes). Of the patients, $449(68.7 \%)$ received a prescription for one or more antibiotics. A diagnosis was specified in 545 of the 654 cases (83.3\%), and in 109 (16.7\%) the diagnosis was unknown. Of the 545 patients with a specified diagnosis, $387(71.0 \%)$ were prescribed an antibiotic, and $243(62.8 \%)$ of these antibiotic prescriptions were non-adherent. Of the 109 patients with no diagnosis, 47 (43.1\%) had no antibiotics prescribed and $62(56.9 \%)$ were prescribed an antibiotic. However, as defined under 'Methods', all prescriptions or non-prescriptions of antibiotics in the group in which the diagnosis was not specified were deemed non-adherent.

\section{Outcome 1: Adherence to guidelines}

Overall, only $45.1 \%$ of antibiotic prescriptions and non-prescriptions in the 654 cases were adherent to guidelines (95\% confidence interval (CI) 41.46 - 49.12). Of the 449 cases in which the patient did receive an antibiotic prescription, $32.1 \%$ were adherent to guidelines (95\% CI 27.90 - 36.55). For the 205 patients who did not receive an antibiotic prescription, adherence was $74.2 \%$ (95\% CI 67.77 - 79.72). If an antibiotic was prescribed, it was significantly less likely to be adherent to guidelines than when an antibiotic was not prescribed (odds ratio (OR) $0.17,95 \%$ CI $0.12-0.24 ; p<0.001$ ).

The majority of the study population was female (61.8\%), adult (77.4\%), and seen by a nurse (64.7\%). Only 6 patients $(0.9 \%)$ were seen by a dentist. Univariate logistic regression analysis showed that none of these variables significantly affected overall guideline

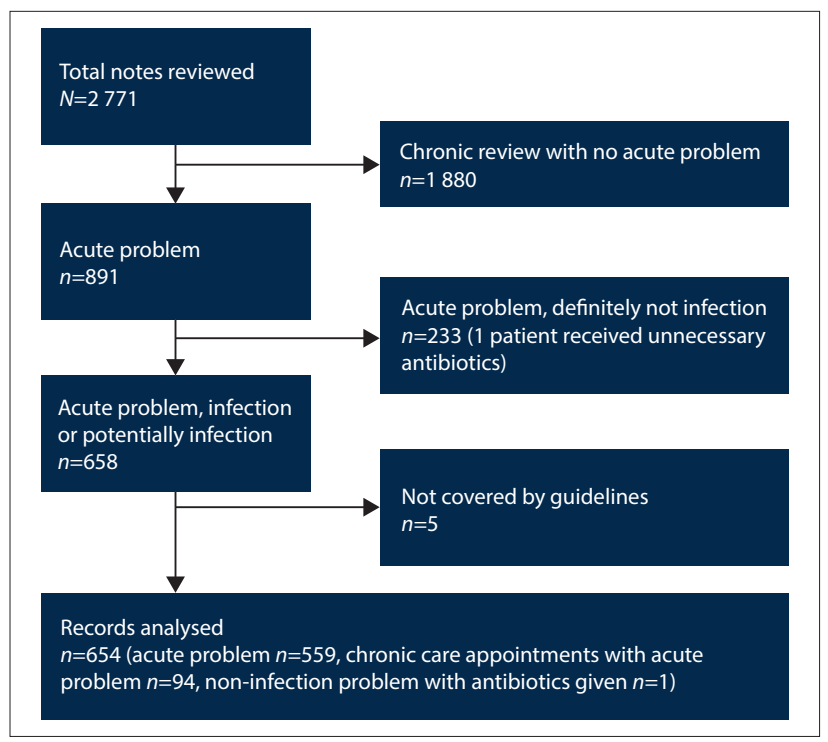

Fig. 1. Inclusion and exclusion of patient records. 
adherence. Multivariate logistic regression analysis suggested that prescriptions for adults were more likely to be adherent to guidelines than those for paediatric patients (Table 1).

The number of patients eligible for inclusion varied from 47 at clinic $\mathrm{H}$ to 125 at clinic A. Adherence varied from $32.8 \%$ at clinic D to $64.9 \%$ at clinic E. Clinic A was used as the reference for logistic regression analysis. Clinics $\mathrm{E}, \mathrm{F}$ and $\mathrm{H}$ were all significantly more likely to be adherent to guidelines than clinic A. These factors retained their significance in the multivariate analysis, with clinic $\mathrm{C}$ also demonstrating improved guideline adherence (Table 1).

The seven most common systems treated were respiratory (27.8\%), dermatology (23.5\%), gastroenterology (7.8\%), urology (7.5\%), sexually transmitted diseases (STDs) (6.9\%), throat (4.1\%) and ears $(5.5 \%)$. The proportion of patients receiving an antibiotic in each physiological system category varied from $100 \%$ for STDs to $13.7 \%$ for gastrointestinal diagnoses. Adherence to guidelines varied significantly between the groups. Dermatology had the highest proportion of cases adherent to guidelines (66.0\%) and was used as the reference for logistic regression analysis. Respiratory, throat and urology cases were all significantly less likely to be adherent to guidelines than dermatology cases. This significance was retained in the multivariate analysis for respiratory and urology cases, but not for throat (Table 1).

The gastrointestinal system had the highest proportion of unknown diagnoses (31.3\%), and STDs the lowest (0\%).

Fourteen different antibiotics were prescribed for the whole cohort of patients reviewed in this study. Of the total of 449 patients who received antibiotics, 370 received a prescription for one antibiotic and 79 a prescription for two or more. The most commonly prescribed antibiotics were amoxicillin (37.9\%), flucloxacillin (12.7\%), metronidazole $(10.9 \%)$, azithromycin $(10.4 \%)$, ceftriaxone $(9.5 \%)$, ciprofloxacin (8.4\%) and phenoxymethylpenicillin (3.0\%). The antibiotics for which most prescriptions adhered to the guidelines were metronidazole (58.3\%), azithromycin $(55.2 \%)$ and ceftriaxone

Table 1. Adherence to guidelines according to different variables with univariate and multivariate logistic regression comparing the odds of being adherent to guidelines compared with reference

\begin{tabular}{|c|c|c|c|c|c|c|c|c|}
\hline \multirow[b]{2}{*}{ Factor } & \multirow[b]{2}{*}{ Total, $N$} & \multirow[b]{2}{*}{ Adherent, $n(\%)$} & \multicolumn{3}{|c|}{ Univariate logistic regression } & \multicolumn{3}{|c|}{ Multivariate logistic regression } \\
\hline & & & OR & CI & $p$-value & OR & CI & $p$-value \\
\hline \multicolumn{9}{|l|}{ Facility } \\
\hline Clinic A & 125 & $42(33.6)$ & Ref & & & Ref & & \\
\hline Clinic B & 87 & $29(33.3)$ & 0.99 & $0.55-1.76$ & 0.97 & 1.80 & $0.88-3.71$ & 0.11 \\
\hline Clinic C & 91 & $37(40.7)$ & 1.35 & $0.77-2.37$ & 0.29 & 2.03 & $1.05-3.96$ & $0.04^{*}$ \\
\hline Clinic D & 58 & $19(32.8)$ & 0.96 & $0.49-1.87$ & 0.91 & 0.94 & $0.44-2.02$ & 0.88 \\
\hline Clinic E & 57 & $37(64.9)$ & 3.66 & $1.89-7.06$ & $<0.001^{\star *}$ & 4.87 & $2.24-10.58$ & $<0.001^{* *}$ \\
\hline Clinic F & 116 & $71(61.2)$ & 3.23 & $1.91-5.48$ & $<0.001^{\star *}$ & 7.91 & $3.96-15.86$ & $<0.001^{* *}$ \\
\hline Clinic G & 73 & $35(47.9)$ & 1.82 & $1.01-3.28$ & 0.47 & 2.21 & $1.07-4.58$ & 0.03 \\
\hline Clinic $\mathrm{H}$ & 47 & $25(53.2)$ & 2.25 & $1.13-4.45$ & $0.02^{*}$ & 4.16 & $1.84-9.43$ & $<0.001^{* *}$ \\
\hline \multicolumn{9}{|l|}{ Patient age group } \\
\hline Paediatric & 148 & $62(41.9)$ & Ref & & & Ref & & \\
\hline Adult & 506 & $233(46.0)$ & 1.19 & $0.82-1.73$ & 0.35 & 1.86 & $1.13-3.08$ & $0.02^{*}$ \\
\hline \multicolumn{9}{|l|}{ Patient gender } \\
\hline Male & 250 & $138(55.2)$ & Ref & & & Ref & & \\
\hline Female & 404 & $221(54.7)$ & 1.03 & $0.75-1.41$ & 0.85 & 0.95 & $0.65-1.40$ & 0.80 \\
\hline \multicolumn{9}{|l|}{ Clinician type } \\
\hline Nurse & 423 & $192(45.4)$ & Ref & & & & & \\
\hline Doctor & 225 & $99(44.0)$ & 0.93 & $0.67-1.29$ & 0.69 & 1.19 & $0.75-1.87$ & 0.46 \\
\hline Dentist & 6 & $4(66.6)$ & 2.38 & $0.43-13.2$ & 0.32 & 22.3 & $2.87-173.9$ & $0.003^{*}$ \\
\hline \multicolumn{9}{|l|}{ Physiological system } \\
\hline Dermatology & 154 & $101(65.6)$ & Ref & & & Ref & & \\
\hline Respiratory & 182 & $62(34.1)$ & 0.27 & $0.17-0.44$ & $0.001^{\star *}$ & 0.39 & $0.21-0.73$ & $0.003^{*}$ \\
\hline Gastroenterology & 51 & $32(62.8)$ & 0.88 & $0.46-1.71$ & 0.71 & 0.62 & $0.28-1.36$ & 0.23 \\
\hline Urology & 49 & $8(16.3)$ & 0.1 & $0.05-0.23$ & $<0.001^{\star *}$ & 0.09 & $0.03-0.28$ & $<0.001^{* *}$ \\
\hline STD & 45 & $29(64.4)$ & 0.95 & $0.48-1.91$ & 0.89 & 1.10 & $0.17-6.98$ & 0.92 \\
\hline Ears & 36 & $18(50.0)$ & 0.52 & $0.25-1.09$ & 0.09 & 1.27 & $0.51-3.21$ & 0.61 \\
\hline Throat & 27 & $10(37.0)$ & 0.31 & $0.13-0.72$ & $0.007^{\star}$ & 0.43 & $0.11-1.71$ & 0.23 \\
\hline \multicolumn{9}{|l|}{ Antibiotic } \\
\hline Amoxicillin & 212 & $53(25.0)$ & 0.27 & $0.19-0.39$ & $<0.001^{\star *}$ & 0.16 & $0.09-0.27$ & $<0.001^{\star *}$ \\
\hline Azithromycin & 58 & $32(55.2)$ & 1.55 & $0.90-2.66$ & 0.11 & 0.48 & $0.18-1.32$ & 0.16 \\
\hline Ceftriaxone & 54 & $29(53.7)$ & 1.45 & $0.83-2.53$ & 0.19 & 0.52 & $0.13-2.08$ & 0.35 \\
\hline Ciprofloxacin & 46 & $8(17.4)$ & 0.23 & $0.11-0.51$ & $<0.001^{\star *}$ & 0.38 & $0.13-1.09$ & 0.07 \\
\hline Flucloxacillin & 72 & $38(52.8)$ & 1.40 & $0.86-2.29$ & 0.18 & 0.40 & $0.20-0.81$ & $0.01^{\star}$ \\
\hline Metronidazole & 60 & $35(58.3)$ & 1.79 & $1.04-3.06$ & $0.04^{*}$ & 2.31 & $0.88-6.05$ & 0.09 \\
\hline Phenoxymethylpenicillin & 17 & $7(41.2)$ & 0.84 & $0.32-2.24$ & 0.73 & 0.30 & $0.06-1.42$ & 0.13 \\
\hline
\end{tabular}


(53.7\%), while the antibiotic with the smallest proportion of prescriptions adhering to guidelines was ciprofloxacin (17.4\%) (Table 1). The antibiotics prescribed for the largest proportions of unknown diagnoses were amoxicillin (16.5\%) and ciprofloxacin (17.0\%).

\section{Outcome 2: Reasons for non- adherence}

The treatment of 357 patients $(54.6 \%$ of the total study population) was not adherent to EML guidelines. ${ }^{[20]}$ Of these cases, $92.7 \%$ were due to a single error and $7.3 \%$ to a combination of errors. The five main errors were (as percentages of the 357 cases in which an error was made) unknown diagnosis (30.5\%), antibiotic not required (21.6\%),

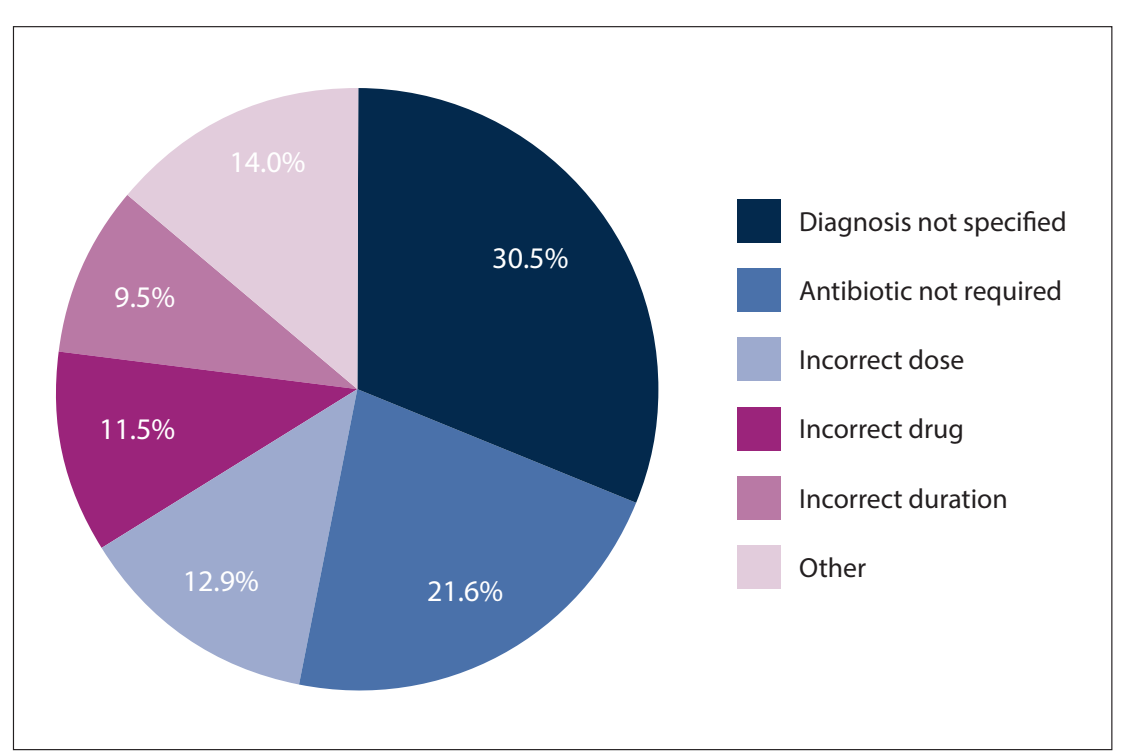

Fig. 2. Reasons for non-adherence to guidelines, as percentages of all cases in which an error was made $(\mathrm{N}=357)$. incorrect dose (12.9\%), incorrect antibiotic choice (11.5\%), and incorrect duration of prescription (9.5\%) (Fig. 2). Of the total study population who received an antibiotic ( $n=449), 17.1 \%$ did not require one.

Of the patients who received an antibiotic, $14.0 \%$ did not have a documented diagnosis, while $22.9 \%$ of those who did not receive an antibiotic did not have a documented diagnosis. If an antibiotic was prescribed, it was significantly less likely to be for an unknown than for a known diagnosis (OR $0.54,95 \%$ CI $0.35-0.82 ; p=0.004$ ).

Of the study population, 205 patients did not receive an antibiotic; in this group, 151 prescriptions $(73.7 \%)$ were adherent to the guidelines, 48 (23.4\%) were not adherent because a diagnosis was not

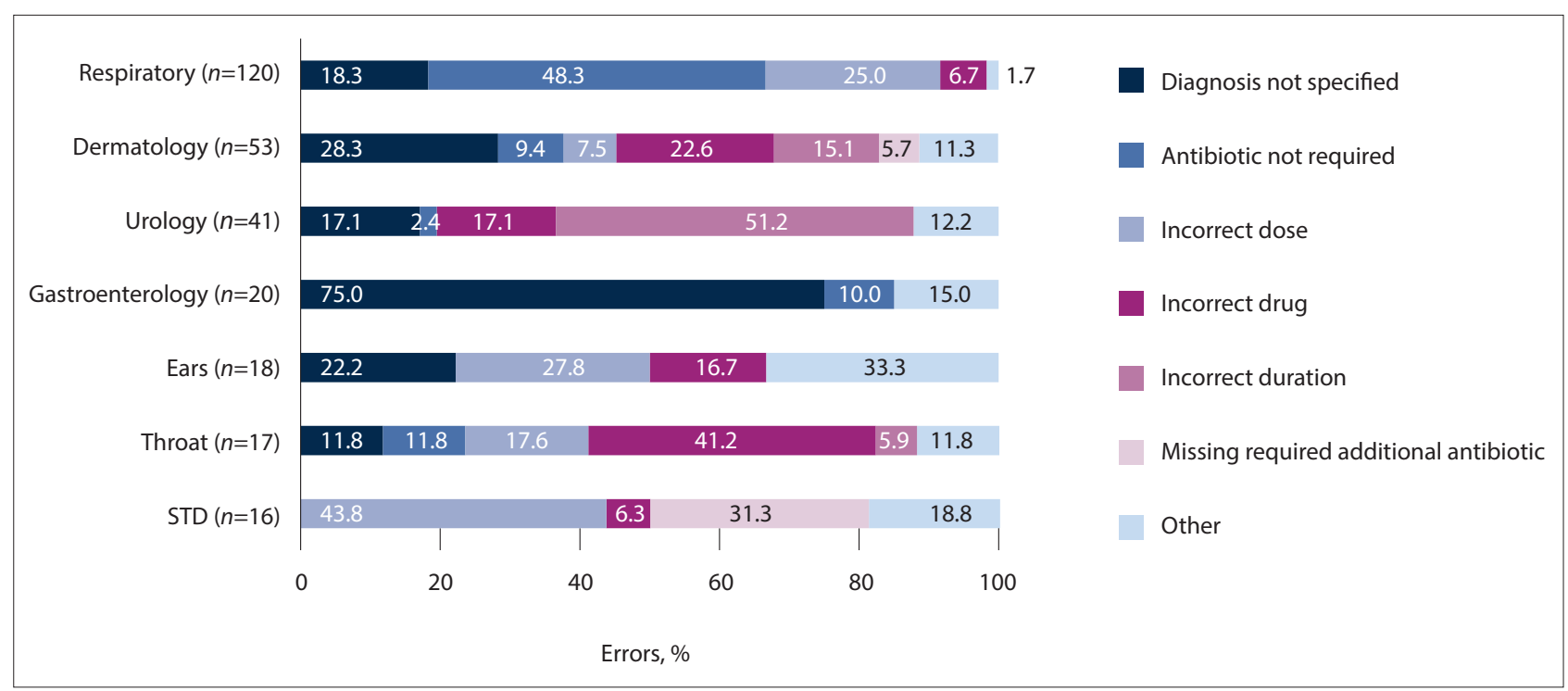

Fig. 3. Reasons for non-adherence to guidelines, according to the seven most common physiological systems. (STD = sexually transmitted disease.) 


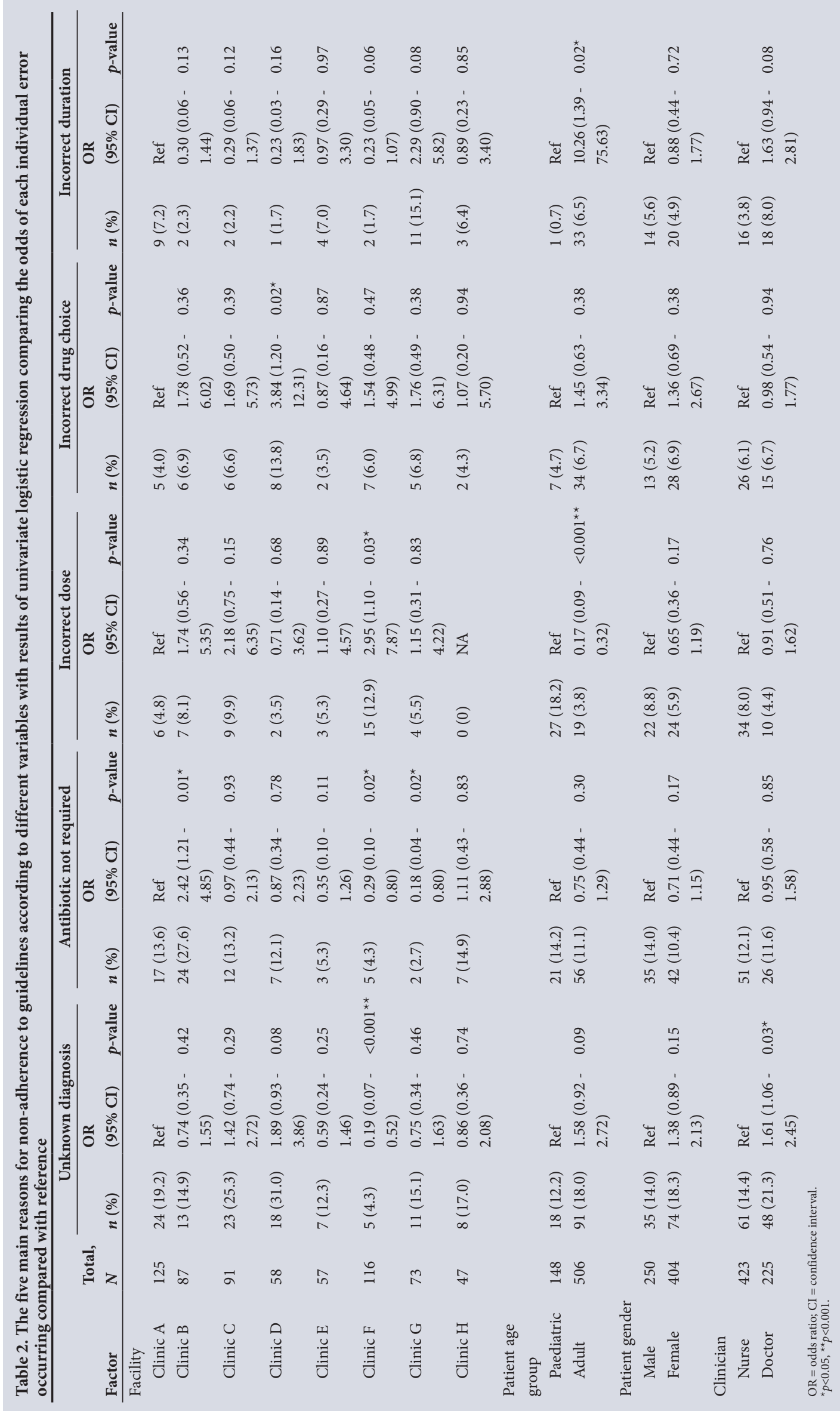


(51.2\%). For dermatology cases, the main errors were not having a diagnosis specified $(28.3 \%)$ and incorrect choice of antibiotic $(22.6 \%)$.

\section{Discussion}

\section{Overall adherence}

Antibiotic resistance is a global problem significantly associated with inappropriate use and overuse of antibiotics. ${ }^{[1-3]}$ This study has demonstrated that in the Cape Town Metro district overall adherence to national guidelines for antibiotic prescribing in primary care was low at only $45.1 \%$. This level contrasts with the results from the WHO pilot study in Brits, SA, where overall compliance with guidelines was found to be $67 \%{ }^{[17]}$ However, in Brits tuberculosis treatment was included and there was no evaluation of appropriate non-prescribing of antibiotics.

The study population of patients with a definite or potential infection can be divided into those who received an antibiotic prescription and those who did not. This allows for comparison of appropriate prescribing and non-prescribing of antibiotics. When an antibiotic was prescribed, adherence to guidelines was a relatively low $32.1 \%$, whereas when an antibiotic was not prescribed adherence was much higher at $74.2 \%$. This indicates that the main problem is with the antibiotic prescriptions themselves rather than cases of infection in which an antibiotic was not prescribed.

\section{Factors affecting adherence}

Multivariate analysis showed that prescribing for adults was more likely to be adherent to guidelines than prescribing for paediatric patients, which may have been due to errors in calculating paediatric doses or lack of experience in paediatric medicine. There was no association with gender or whether the healthcare professional was a doctor or a nurse. This is an important finding for the development of a targeted intervention.

\section{Facility}

We found a significant difference in levels of adherence between different facilities, so clinics performing poorly should be targeted for interventions first and those performing better could be analysed further to understand why they are more likely to have appropriate prescribing practices. Other studies have suggested that the socioeconomic circumstances in the area in which the facility is situated affect adherence. ${ }^{[8]}$ This aspect requires further evaluation and did not form part of our study.

\section{Physiological system and type of antibiotic}

Guideline adherence varied between physiological systems and according to which antibiotic was prescribed, which can also be used to guide the development of targeted interventions. A positive finding of the study was that the physiological system that had the highest adherence rates was dermatology (65.6\% adherent). The main reasons for error were because a diagnosis was not specified (28.3\%) and use of the incorrect drug (22.6\%).

Although it is appropriate that all patients diagnosed with an STD received an antibiotic, only $64.4 \%$ of these prescriptions adhered to guidelines. This was the second most adherent physiological group, and this finding relates to the fact that the antibiotics with the highest levels of adherence were metronidazole, azithromycin and ceftriaxone, all commonly used to treat STDs at primary care level. STDs was the only system that had no unknown diagnoses, probably because the STD proformas used by facilities require a diagnosis to be encircled.
The appropriately low antibiotic prescription rate for patients presenting with gastrointestinal problems is a positive finding. They had the lowest proportion of antibiotics prescribed (13.7\%). A common complaint in this group is acute diarrhoea, and in contrast, international studies have shown an inappropriately high level of prescribing for this group. ${ }^{[10]}$ However, patients with gastrointestinal problems had the highest proportion of unknown diagnoses (31.4\%), which may indicate diagnostic uncertainty in this area.

The system that had the lowest adherence rates was the urological system, and the antibiotic with the lowest adherence rate was ciprofloxacin, most commonly prescribed for urinary tract infections (UTIs). When evaluating why prescribing for urological problems did not adhere to guidelines, the most common reason was incorrect duration of treatment (Fig. 3). International studies reveal a range in guideline adherence for UTIs from $22.2 \%$ in Slovenia to $72.7 \%$ in The Netherlands. ${ }^{[12]}$ There are also findings from other countries that incorrect duration of antibiotic treatment for UTIs is a common problem. One study showed that $82 \%$ of trimethoprim prescriptions, $73 \%$ of nitrofurantoin prescriptions and $71 \%$ of fluoroquinolone prescriptions for UTIs were prescribed for longer periods than guidelines recommended. ${ }^{[11]}$

For patients with respiratory problems, only $34.1 \%$ of cases were adherent to guidelines. Of those that were not adherent, $48.3 \%$ of the errors were because the antibiotic was not required (Fig. 3). This is consistent with international findings; for example, a Malaysian study found that $31.8 \%$ of prescriptions were unnecessary, ${ }^{[9]}$ and a study in Botswana found that $79 \%$ of prescriptions were unnecessary. ${ }^{[10]}$ These results are valuable in that they have identified another area of targeted intervention, since many of these cases are of viral origin.

The difference between adherence in different physiological systems is important, as it identifies areas that could potentially be targeted for education campaigns and further research.

\section{Reasons for non-adherence}

This is the first study in SA primary healthcare that has looked at the reasons for non-adherence to guidelines. The commonest reason was because a diagnosis was not specified in the medical records. What is unknown in this group is whether the clinician did make a diagnosis but did not record it, or there was true diagnostic uncertainty. As we did not interview the clinicians, we are unable to elucidate this aspect further, and we could have underestimated the actual adherence to guidelines. This is a study limitation and requires further research. It should be noted that in this study, lack of recording of a diagnosis was determined to be a significant reason for non-adherence and so was included in the analysis. Failure to record a diagnosis has medicolegal implications and is an important area that must be addressed. Doctors were significantly more likely than nurses not to specify a diagnosis.

The second most common error was that the antibiotic was not required. This applied to $17.1 \%$ of all cases in which an antibiotic was prescribed. This irrational use of antibiotics is associated with the development of antibiotic resistance and is a potential cause of adverse effects.

The third most common error was prescribing the incorrect dose (12.9\%), particularly for urology cases (Fig. 3). Paediatric patients were significantly more likely than adult patients to receive an incorrect dose. Paediatric doses are usually adjusted by weight, requiring complicated calculations that may have contributed to the error in this group. Other reasons were use of the incorrect drug (11.5\%) and prescribing the medication for the incorrect 
duration (9.5\%). Once again, these errors highlight areas for targeted intervention and education.

Of the 205 cases in which an antibiotic was not prescribed, 73.7\% were adherent, $23.4 \%$ were non-adherent because a diagnosis was not specified, and $2.9 \%$ were non-adherent because an antibiotic should have been prescribed. Again, this finding demonstrates the significant implications of not specifying a diagnosis, and that despite a general pattern of overprescribing there were six cases where an antibiotic was not prescribed when it should have been.

\section{Study strengths and limitations}

This study demonstrated poor antibiotic guideline adherence, with additional details on why antibiotics were prescribed and associations with specific variables. Reasons why prescriptions were not adherent to guidelines have not been published previously in SA. The methodology was standardised and robust, with an objective comparison of prescriptions to current national guidelines for primary care.

Limitations are that the study evaluated prescribing data only and did not confirm whether the correct diagnosis was made. This may have led to underestimation of the level of inappropriate prescribing.

Clinical decision-making is complex, and prescribing in certain clinical scenarios may not be conducive to following guidelines. Clinical experience and judgements were not considered in this study.

The study also did not include prophylactic antibiotic use or the use of antituberculosis medication. It is therefore an insight into only one aspect of all antibiotic use.

\section{Recommendations}

The study findings revealed an urgent need to address antibiotic prescribing practice in primary care. It suggests the need for targeted education of both healthcare professionals and patients to better understand rational antibiotic prescribing. There should be increased use and accessibility of technology, including guideline and prescribing phone apps, and also empowerment of the pharmacist to act as gatekeeper to ensure appropriate and correct prescribing. Further studies should include qualitative assessments of healthcare workers' knowledge, attitudes and antibiotic prescribing practices, as well as use of clinical guidelines.

\section{Conclusion}

Guidelines provide an evidence-based framework for the appropriate prescribing of antibiotics that is important for reducing the risk of antibiotic resistance. This study shows that despite the availability of published national guidelines, adherence to these in the Cape Town Metro District is low.

The study identified significant differences in adherence to guidelines between facilities, and according to the physiological system being treated and the antibiotics being used. There was a wide range of reasons for non-adherence to guidelines, but importantly almost $20 \%$ of all patients who received an antibiotic prescription did not require the antibiotic. This irrational prescribing compounds the risk of antibiotic resistance and the potential for adverse events.

Importantly, the high proportion of healthcare professionals who did not document a diagnosis, which is critical to rational prescribing, prevented a complete understanding of the problem.

Poor antibiotic prescribing practice is associated with an increased potential for antibiotic resistance. This study has shown an urgent need to address antibiotic prescribing in primary care in the Cape Town Metro District.
Acknowledgements. We thank Improving Global Health, funded by THET/UK Aid in association with Health Education England and the NHS Thames Valley and Wessex Leadership Academy, the Health Impact Assessment Directorate of the Western Cape Department of Health, and the University of Cape Town School of Public Health and Family Medicine for supporting this project and enabling the research to be carried out. We also thank the staff at the participating CDCs and CHCs for facilitating data collection.

Author contributions. JG developed the study protocol, collected and analysed data and wrote the manuscript. BW and MB contributed to the design of the study, data analysis and reviewing and editing the manuscript.

Funding. JG's work was supported by THET/UK Aid in association with Health Education England and the NHS Thames Valley and Wessex Leadership Academy. There was no other external funding.

Conflicts of interest. None.

1. World Health Organization. Antimicrobial Resistance, Global Report on Surveillance: Summary. 2014 http://apps.who.int/iris/bitstream/10665/112647/1/WHO_HSE_PED_AIP_2014.2_eng.pdf?ua=1 (accessed 20 June 2016)

2. O’Neill J. Tackling drug-resistant infections globally: Final report and recommendations. The review on antimicrobial resistance. May 2016. https://amr-review.org/sites/default/files/160518_Final\%20paper with\%20cover.pdf (accessed 16 March 2018).

3. Goossens $\mathrm{H}$, Ferech M, vander Stichele R, et al. Outpatient antibiotic use in Europe and association with resistance: A cross-national database study. Lancet 2005;365(9459):579-587. https://doi.org/10.1016/ S0140-6736(05)17907-0

4. Costelloe $\mathrm{C}$, Metcalfe $\mathrm{C}$, Lovering $\mathrm{A}$, et al. Effect of antibiotic prescribing in primary care on antimicrobial resistance in individual patients: Systematic review and meta-analysis. BMJ 2010;340:c2096. https://doi. org/10.1136/bmj.c2096

5. National Department of Health, South Africa. Antimicrobial Resistance: National Strategy Framework National Department of Health, South Africa. Antimicrobial Resistance: National Strategy Framework
2014 - 2024. 2014. http://www.mm3admin.co.za/documents/docmanager/3C53E82B-24F2-49E1-B9972014 - 2024. 2014. http://www.mm3admin.co.za/docum

6. Department of Health, UK. The Path of Least Resistance, Standing Medical Advisory Committee, SubGroup of Antimicrobial Resistance. 1997. http://antibiotic-action.com/wp-content/uploads/2011/07/ Standing-Medical-Advisory-Committee-The-path-of-least-resistance-1998.pdf (accessed 20 June 2016). 7. Ivanovska V, Hek K, Mantel Teeuwisse AK, et al. Antibiotic prescribing for children in primary care and adherence to treatment guidelines. J Antimicrob Chemother 2016;71(6):1707-1714. https://doi. org/10.1093/jac/dkw030

8. Dallas A, Magin P, Morgan S, et al. Antibiotic prescribing for respiratory infections: A cross-sectional analysis of the ReCEnT study exploring the habits of early-career doctors in primary care. Fam Pract 2015;32(1):49-55. https://doi.org/10.1093/fampra/cmu069

9. Rezel RS, Hassali MA, Alrasheedy AA, et al. Prescribing patterns for upper respiratory tract infections: A prescription review of primary care practice in Kedah, Malaysia, and the implications. Expert Rev Anti A prescription review of primary care practice in Kedah, Malaysia, and the implication
Infect Ther 2015;13(12):1547-1556. https://doi.org/10.1586/14787210.2015.1085303

10. Boonstra $E$, Lindbaek $M$, Ngome $E$. Adherence to management guidelines in acute respiratory infections and diarrhoea in children under 6 years old in primary health care in Botswana. Int $J$ Qual Health Care
and and diarrhoea in children under 6 years old in primary heal

11. Grigoryan L, Zoorob R, Wang H, Trautner BW. Low concordance with guidelines for treatment of acute cystitis in primary care. Open Forum Infect Dis 2015;2(4):ofv159. https://doi.org/10.1093/ofid/ofv159

12. Philips H, Huibers L, Holm Hansen E, et al. Guidelines adherence to lower urinary tract infection treatment in out-of-hours primary care in European countries. Qual Prim Care 2014;22(4):221-231. http://primarycare.imedpub.com/guidelines-adherence-to-lower-urinary-tract-infection-treatment-inoutofhours-primary-care-in-european-countries.pdf (accessed 5 March 2018).

13. Rico-Ferreira P, Palazon-Bru A, Calvo-Perez M, Gil-Guillen VF. Nonadherence to guidelines for prescribing antibiotic therapy to patients with tonsillitis or pharyngotonsillitis: A cross sectional study. Curr Med Res Opin 2015;31(7):1319-1322. https://doi.org/10.1185/03007995.2015.1041896

14. Tell D, Engstrom S, Molstad S. Adherence to guidelines on antibiotic treatment for respiratory tract infections in various categories of physicians: A retrospective cross-sectional study of data from electronic patient records. BMJ 2015;5:e008096. https://doi.org/10.1136/bmjopen-2015-008096

15. Teixeria Rodrigues A, Ferreira M, Pineiro-Lamas M, et al. Determinants of physician antibiotic prescribing behaviour: A 3 year cohort study in Portugal. Curr Med Res Opin 2016;32(5):949-957.

16. Truter, I. Antimicrobial prescribing in South Africa using a large pharmacy database: A drug utilisation Truter, I. Antimicrobial prescribing in South Africa using a large pharmacy database: A drug
study. S Afr J Infect Dis 2015;30(2):52-56. https://doi.org/10.1080/23120053.2015.1054181

17. World Health Organization. Community-based Surveillance of Antimicrobial Use and Resistance in
17. S Afr Infect Dis 2015;30(2):52-56. https:/doi.org/10.1080/23120053.2015.1054181 Resource-constrained Settings. 2009. http://www.who.int/medicines/publications/community_based_ may09.pdf?ua $=1$ (accessed 21 June 2016).

18. Katende-Kyenda NL, Lubbe MS, Serfontein JHP, Truter I. Antimicrobial prescribing patterns in a group of private primary health care clinics in South Africa. Health SA 2007;12(1):21-29. https://doi. org/10.4102/hsag.v12i1.240

19. World Health Organization, Food and Agriculture Organization of the United Nations, World Organisation for Animal Health. Antimicrobial Resistance: A Manual for Developing National Action Plans. 2016. http://apps.who.int/iris/bitstream/10665/204470/1/9789241549530_eng.pdf (accessed 20 June 2016).

20. National Department of Health, South Africa. Essential Drugs Programme. Standard Treatment Guidelines and Essential Medicines List for South Africa, Primary Healthcare Level, 2014 edition. 2014. Guidelines and Essential Medicines List for South Africa, Primary Healthcare Leve,
http://www.kznhealth.gov.za/pharmacy/edlphc2014a.pdf (accessed 20 June 2016).

21. Western Cape Government Health. Annual Performance Plan 2016 - 2017. 2016. https://www. westerncape.gov.za/assets/departments/health/app2016-2017.pdf (accessed 20 June 2016). 